\title{
Hybrid Graphene/Au ActivatableTheranostic Agent for Multimodalities Imaging Guided Enhanced Photothermal Therapy
}

\author{
Gao Shi ${ }^{1, \dagger}$, Liwen Zhang ${ }^{2, \dagger}$, Guohao Wang ${ }^{2}$, Kai Yang ${ }^{3}$, Minglong Chen ${ }^{1}$, Rui Tian ${ }^{4}$, Qingjie \\ $\mathrm{Ma}^{1, *}$, Lei $\mathrm{Zhu}^{2, *}$
}

1. Department of Nuclear Medicine, China-Japan Union Hospital, Jilin University, Changchun, Jilin 130033, P. R. China.

2. State Key Laboratory of Molecular Vaccinology and Molecular Diagnostics \& Center for Molecular Imaging and Translational Medicine, School of Public Health, Xiamen University, Xiamen 361005, China;

3. School of Radiation Medicine and Protection \& School for Radiological and Interdisciplinary Sciences (RAD-X), Collaborative Innovation Center of Suzhou Nano Science and Technology \& Collaborative Innovation Center of Radiation Medicine of Jiangsu Higher Education Institutions, Medical College of Soochow University, Suzhou, Jiangsu 215123, China;

4. Department of Ophthalmology Second Hospital, Jilin University, Changchun, Jilin 130033, P. R. China.

$\uparrow$ These authors contributed equally to this work.

*To whom correspondence should be addressed. Tel: (+)86-592-2880642, Fax: (+)86-5922880642; E-mail:lei.zhu@xmu.edu.cn(L.Z.) and maqingjiejlu@163.com (Q. M.). 


\section{ABSTRACT}

Photothermal therapy (PTT) has been increasingly investigated. However, there are still challenges in strategies that can further enhance photoconversion efficiency and improve photothermal tumor ablation effect of current nanomaterials.Herein, we developed a fluorescent/photoacoustic imaging guided PTT agent by seeding Gold (Au) nanoparticles onto graphene oxide (GO). Near infrared dye (Cy5.5) labeled-matrix metalloproteinase-14 (MMP-14) substrate (CP) was conjugated onto the $\mathrm{GO} / \mathrm{Au}$ complex (GA) forming tumor targeted theranostic probe (CPGA), whereCy5.5 fluorescent signal is quenched bySurface Plasmon Resonance (SPR) capacity from both GO and Au, yet itcanboost strong fluorescence signals after degradation by MMP-14. The photothermal effect of GA hybrid was found significantly elevated compared with $\mathrm{Au}$ or GO alone. After intravenous administration of CPGA into SCC7 tumorbearing mice, high fluorescence and PA signals were observed in the tumor area over time, which peaked at the $6 \mathrm{~h}$ time point (tumor-to-normal tissue ratio of $3.64 \pm 0.51$ for optical imaging and $2.5 \pm 0.27$ for PA imaging). The tumors were then irradiated with a laser, and an excellent tumor inhibition was observedwithoutrecurrence. Our studies further encourage applications of the hybrid nanocomposite for image-guided enhanced PTT in biomedical applications, especially in cancer theranostics.

KEYWORDS: image-guided therapy, activatable probes, graphene oxide, photothermal therapies, matrix metalloproteinase

\section{Introduction}


Photothermal therapy (PTT), by which nanomaterials ablate cancer cells or tissues through conversion of near-infrared (NIR) light into thermal energy, has been increasingly investigated[16]. Due to their large surface area per particle and excellent light absorption in the NIR range [7, 8], various nanomaterials such as gold $(\mathrm{Au})$ nanoparticles, carbon nanotubes and graphenes are preclinically investigated as desirable agents for photothermal treatment of diseases, especially tumors. However, there are still challenges in strategies that can further enhance photoconversion efficiency and improve photothermal tumor ablation effect of current nanomaterials with reduced damaging of normal cells and tissues.

Graphene and its derivatives constitute a new type of agent in the field of biomedicine. In the view of its special surface properties, excellent photo-thermal conversion efficiency and the potential for extra engineering, graphene often serves as a platform for facilitating tumor diagnosis or therapeutics by incorporation of imaging probes, drugs or other nanomaterials via both covalent and non-covalent conjugation methods. For example, varieties of inorganic materials have been ligated on the surface of graphene or its derivatives, producing a hybrid nanocomplex for broad spectrum applications in antibiosis[9], catalysis[10], multi-modalities imaging-guided therapy[11, 12], gene/drug delivery[9, 13-16]. Thus, the development of graphene-based hybrid nanocomplexes can potentially improve photothermal ablation. Optimization of treatment schedules is another key factor that can improve PTT efficiency of nanomaterials. Ideally, PTT is more effective when the PTT agent prominently accumulates in the tumor. With the development of molecular imaging, it is now possible to guide PTT strategies by single or multi-modalities imaging techniques. Fluorescence imaging and photoacoustic (PA) imaging are usually employed in the field of image-guided therapy due to their low cost, low radiation risk, high sensitivity and translational potency[17-24]. Many groups, including ours, 
have reported an effective ablation of tumors with the use of fluorescent/PA imaging techniques[24-26], demonstrating the potential of applying imaging technique for tumor-targeted therapy. Even though successful studies have aimed to provide improved PTT effects by either constructing hybrid nanocomplexes or utilizing multimodalities image-guided therapy platforms, to the best of our knowledge, applications on the development a tumor microenvironment (TME)-targeted probe possessing both high imaging sensitivity and enhanced PTT effect have been rarely reported.

Herein, we present an improved smart theranostic probe (CPGA) based on graphene oxide (GO)-Au nanoparticle nanocomposite (GA), which was covalently functionalized with an NIR dye-labeled matrix metalloproteinase-14 (MMP-14 or MT1-MMP) peptide substrate (CP) [27, 28], for applications in fluorescent/PA image-guided enhanced PTT of tumors (Scheme 1). Au nanoparticles at diameter of $15 \pm 1.6 \mathrm{~nm}$ were ligated on the surface of GO due to their excellent photothermal property as a PTT agent and Surface Plasmon Resonance (SPR) capacity as a fluorescent quencher[29, 30]. Specifically, CPGA remains intact and thus maintains its quenched state[31], while it boosts strong fluorescence signals after degradation by MMP-14, a key endopeptidase that is overexpressed on tumor cell surface[32], thus providing real-time images of CPGA from whole body. Meanwhile, the combined strong photon absorbance of the Au/Go complex allows enhanced PA signals compared to single compositions. Under the guidance of optical imaging and PA imaging that locally analyzes CPGA behaviors at the tumor site; CPGAmediated in vivo PTT was carried out. We observed that the tumors were efficiently ablated after $808 \mathrm{~nm}$ laser irradiation at low density $\left(0.75 \mathrm{~W} \mathrm{~cm}^{-2}\right)$ in mice that received CPGA intravenously (i.v). Compared to the other activatable probes, synthetic CPGA had multiple advantages: (i) CPGA simultaneously provided enzyme triggered fluorescent/PA imaging and effective tumor 
therapy for precise tumor theranosticsvia a simple nanocomplex; (ii) as a hybrid nanocomplex, CPGA, had prominent quenching efficiency (95\%) compared to either CP or CPG alone, which yielded an ultra-sensitive MMP-14-targeted fluorescent signal recovery; and (iii) CPGA exhibited enhanced photoconversionefficiencythan either CP or CPG alone for tumor ablation without recurrence. Overall, our results show remarkable promise for future development of thermo-activatable hybrid nanocomplexes for precise image-guided tumor treatment.

\section{Materials and Methods}

Synthesis of CPGA. MMP-14 sensitive substrate peptide was synthesized as previously reported.[24] In brief, MMP-14 substrate, Gly-Ala-Thr-Arg-Leu-Phe-Gly-Ile-Arg-Gly, was automaticllysynthesized on the Peptide Synthesizer (CS Bio CO, Menlo Park, CA) at 0.1 mmol scale using a Fmoc-Gly-Wang resin. After being released from the resin by $3 \mathrm{~mL}$ TFA cocktail (80\% trifluoroacetic acid (TFA), 10\% thioanisole, 5\% 1,2-Ethanedithiol and 5\% ultrapure water), MMP-14 substrate was precipitated in cold ethyl ether $\left(-20^{\circ} \mathrm{C}\right)$ and lyophilized. The peptide was purified by HPLC using a linear gradient of $20 \%$ to $90 \%$ acetonitrile/water $(0.1 \%$ TFA) for 30 $\min$ at $3.5 \mathrm{~mL} \mathrm{~min}^{-1}$ flow rate, and then lyophilized. The purity was analyzed by analytical HPLC $(\mathrm{C} 18,5 \mu \mathrm{m}, 250 \times 4.6 \mathrm{~mm})$ with $5 \%$ to $65 \%$ acetonitrile containing $0.1 \%$ TFA versus ultrapure water containing $0.1 \%$ TFA over $30 \mathrm{~min}$ at a flow rate of $1 \mathrm{~mL} \mathrm{~min}^{-1}$. The purity was $>90 \%$. Molecular weight of peptide was confirmed by mass spectrum (Thermo Fisher Scientific, Massachusetts, USA) as 1046.60.Next, MMP substrate (10 $\mu \mathrm{M})$ was reacted with an NIR dye (Cy5.5-HNS, $10 \mu \mathrm{M}$ ) in N, N-Dimethylformamide (DMF, $400 \mathrm{~mL}$ ) containing 3\% N, N-Diisopropylethylamine (DIPEA) for $40 \mathrm{~min}$ at $25^{\circ} \mathrm{C}$ in the dark. The Cy5.5 conjugated MMP14 substrate (CP) was purified by HPLC (C18 column, $10 \mu \mathrm{m}, 250 \times 10 \mathrm{~mm})$ in the same condition as described above. The purity was $>90 \%$ as confirmed by analytic HPLC. Molecular 
weight of peptide was confirmed by mass spectrum as 1611.92. After that, $\mathrm{CP}(5 \mu \mathrm{M})$ was reacted with Dipyrrolidino (N-succinimidyloxy) carbeniumhexafluoropho (HSPyU, $5 \mu \mathrm{M}$ ) in $500 \mu \mathrm{L}$ DMF solution containing $3 \%$ of DIPEA for $30 \mathrm{~min}$ to active carboxyl group. Next, excess amount of amine-PEG-amine $(15 \mu \mathrm{M}, 2 \mathrm{kDa})$ was added and stirred overnight in the dark. The following day, final products, CP-PEG-amine, were purified by HPLC (C18 column, $10 \mu \mathrm{m}$, $250 \times 10 \mathrm{~mm}$ ) in the same condition as described above. The purity of CP-PEG-amine was then confirmed by HPLC $(\mathrm{C} 18,5 \mu \mathrm{M}, 250 \times 4.6 \mathrm{~mm})$ yielding $5 \%$ to $65 \%$ acetonitrile containing $0.1 \%$ TFA/ultrapure water containing $0.1 \%$ TFA over $40 \mathrm{~min}$ at a flow rate of $1 \mathrm{~mL}$ min-1.

GA composites were synthesized as previously reported with modifications.[33] Au was seeded onto Graphene Oxide (GO) at the beginning. Briefly, the purified GO (1 mg) was dispersed in $4 \mathrm{~mL}$ of ultrapure water with sonication for $2 \mathrm{~h}$. An aqueous solution of GO (0.25 $\left.\mathrm{mg} \mathrm{mL} \mathrm{mL}^{-1}, 4 \mathrm{~mL}\right)$ was added to $\mathrm{HAuCl}_{4}$ solution $\left(0.47 \mathrm{mg} \mathrm{mL}^{-1}, 10 \mathrm{~mL}\right)$ and stirred for $30 \mathrm{~min}$ to increase the interaction between gold ions and GO. After being heated at $80^{\circ} \mathrm{C}$ and kept for 10 min, a solution of sodium citrate $(0.085 \mathrm{mM}, 200 \mu \mathrm{L})$ was added into the mixed solution and continuous stirred for $2 \mathrm{~h}$. Subsequently, the solution was cooled to room temperature, stirring for overnight. The obtained composite was purified by centrifugation $\left(6000 \mathrm{rmp}, 4^{\circ} \mathrm{C}, 10 \mathrm{~min}\right)$ and washed several times with ultrapure water to remove excess sodium citrate and free gold ions, obtaining the final product $\left(\mathrm{GA}, 1 \mathrm{mg} \mathrm{mL}^{-1}\right)$.

The next day, CP was conjugated onto GO in GA complex. N-(3-dimethylaminopro-pylN'ethylcarbodiimide) hydrochloride (EDC $(0.25 \mu \mathrm{M})$ and N-Hydroxysuccinimide (NHS $(0.25 \mu \mathrm{M})$ were added to the solution of GA nanoparticles $(1 \mathrm{mg})$ at $\mathrm{pH} 7.8$ for activation of the carboxylic groups on GO in GAnanocomplex. 10 min later, CP-PEG-amine $(0.25 \mu \mathrm{M})$ was added into the above solution and stirred overnight at room temperature in the dark, forming CPGA. Next, 
PEG-amine $(0.25 \mu \mathrm{M}, 5 \mathrm{kDa})$ was chemically conjugated to CPGA under the same methods to stabilize the dispersion. The resulting mixture was poured into the ultrafiltration tube $(1.5 \mathrm{~mL})$ and excess CP and PEG was removed by centrifugation at 10,000 rpm and washed using ultrapure water for several times.

Characterization. The nanostructure and size of CPGA were observed by Atomic force microscopy (AFM) (Veeco Instruments Inc, Santa Barbara, USA) and Transmission electron microscopy (TEM) (Bruker, Germany). Transmission electron microscope (TEM) imaging of nanoparticle samples were carried out by using a FEI Tecnai F20 TEM at an acceleration voltage of $200 \mathrm{kV}$. The grids was 400 mesh. Sample solution was dropped to 400 mesh copper network and was dried before characterization. The pressure of column was less than $2.7 \times 10^{-5} \mathrm{~Pa}$. Zeta potentials of MMP substrate, CP, GA and CPGA were measured by dynamic light scattering (DLS) (Varian, Palo Alto, USA). Fluorescent signals of CP, CPG and CPGA were measured using a fluorescence spectrophotometer (Varian, Palo Alto, USA). UV-vis absorbance spectra of CP, Au, GO, GA, CPG and CPGAwereobserverd by Multiskan Go microplate reader (Thermo Fisher Scientific, Massachusetts, USA).

In vitro photoacoustic tomography. CPGA and CPG contain the same amount of GO (499, 250, 125, 62 and $\left.31 \mu \mathrm{g} \mathrm{mL}^{-1}\right), \mathrm{CPGA}$ and $\mathrm{Au}$ contain the same amount of $\mathrm{Au}(501,250,125,62$ and $\left.31 \mu \mathrm{g} \mathrm{mL}^{-1}\right)$ and CPGA, CPG,CP possessed same amount of $\mathrm{CP}\left(50,25,12,6,3 \mu \mathrm{g} \mathrm{mL}^{-1}\right)$ were subjected to PA imaging. CPGA, CPG, CP and Au were put into eppendorf tubes $(1.5 \mathrm{~mL})$ before imaging and all the tubes were immersed at the same depth in the deionized water. Photoacoustic tomography of all samples were captured at $808 \mathrm{~nm}$ using EndraNexus128 (Ann Arbor, MI, USA).

Enzyme activity test. Sensitivity of CPGA to MMP-14 was tested as previously reported. The 
fluorescence of CPGA was measured in TCNB buffer $\left(50 \mathrm{mMTris} \bullet \mathrm{HCl}, 10 \mathrm{mM} \mathrm{CaCl} 2 \cdot 2 \mathrm{H}_{2} \mathrm{O}\right.$, $0.15 \mathrm{M} \mathrm{NaCl}, 0.05 \%$ Brij35, $\mathrm{pH} 7.8$ ) containing $40 \mathrm{nM}$ of activated MMP-14, and incubated in TCNBbuffer at $37^{\circ} \mathrm{C}$ for $120 \mathrm{~min}$. The UV-vis-NIR spectrums were obtained at indicated time points using a fluorescence spectrophotometer. After incubation, Fluorescent images of sample were captured by Carestream FX PRO (Carestream Health Inc., Toronto, CA). The excitation wavelength was set at $675 \mathrm{~nm}$, and the emission spectrum was recorded from 690 to $800 \mathrm{~nm}$ for Cy5.5. Three independent experiments were conducted in parallel.

In vitro photothermal ablation test. The aqueous solutions of $\mathrm{CPGA}, \mathrm{CPG}, \mathrm{Au}$ and $\mathrm{CP}$ containing the same concentration $\left(100 \mu \mathrm{g} \mathrm{mL}^{-1}\right)$ in $1.5 \mathrm{~mL}$ Eppendorf tubes were irradiated by laser ( $808 \mathrm{~nm}$, Stone laser, Shenzhen) at power density of $0.3 \mathrm{~W} \mathrm{~cm}^{-2}$ for $10 \mathrm{~min}$. The laser spot was adjusted to make sure covering the entire surface of samples. Real-time thermal imaging of different solutions was obtained using a FLIR Ax5 camera (FLIR Systems Inc., Wilsonville, USA) and quantified by BM_IR software.

The in vitro photothermal effect and cytotoxicity of CPGA was evaluated by MTT assay on SCC7 cells, which were cultured in Dulbecco's Modified Eagle Medium (DMEM)/ high glucose medium supplemented with $10 \%$ FBS and $1 \%$ antibiotic solution at $37{ }^{\circ} \mathrm{C}$ and $5 \% \mathrm{CO}_{2}$. For in vitro PTT, SCC7 cells at a density of $1 \times 10^{4}$ cells well $^{-1}$ were seeded in a 96-well flat-bottomed plate and incubated overnight. After being respectively incubated CPGA, CPG and GA at indicated concentration for $12 \mathrm{~h}$, cells were washed with PBS for several times and changed the fresh DMEM/ high glucose. Experimental groups (CPGA, CPG and GA) were then exposed to NIR light $\left(808 \mathrm{~nm}, 0.5 \mathrm{~W} \mathrm{~cm}^{-2}\right)$ for $10 \mathrm{~min}$ and incubated for an additional $24 \mathrm{~h}$ at $37^{\circ} \mathrm{C}$. Cells in control groups without the NIR irradiation were incubated under the same conditions. Cell viability was evaluated by MTT assay. 
Photothermal therapy was also investigated by 3'6'-Di (O-acetyl) -4'5'-bis [N, N-bis (carboxymethyl) aminomethyl] fluorescein, tetraacetoxymethyl ester (Calcein AM)/Propidium Iodide (PI) staining (Sangon Biotech, Shanghai, China). SCC7 cells that grown to $80 \%$ confluence in 6-well chambers were incubated with DMEM/ high glucose medium containing CPGA, CPG, CP and PBS at a concentration of $100 \mu \mathrm{g} \mathrm{mL} \mathrm{m}^{-1}$ for overnight, respectively. After being exposed to $808 \mathrm{~nm}$ NIR light at $0.5 \mathrm{~W} \mathrm{~cm}^{-2}$ for 5 minutes, cells were washed cells three times with PBS and added the fresh DMEM/ high glucose medium, incubating for an additional $4 \mathrm{~h}$ at $37^{\circ} \mathrm{C}$, followed by incubation for $4 \mathrm{~h}$ under identical conditions as the control groups in a dark. Removing the cultural medium, cells were added PBS that contained with Calcein AM (4 $\mu \mathrm{M}$, live cells, green fluorescence; $\lambda \mathrm{ex}=490 \mathrm{~nm}, \lambda \mathrm{em}=515 \mathrm{~nm})$ and PI solutions $(4 \mu \mathrm{M}$, dead cells, red fluorescence; $\lambda \mathrm{ex}=490 \mathrm{~nm}, \lambda \mathrm{em}=617 \mathrm{~nm}$ ) and incubated for an additional $25 \mathrm{~min}$ at $37{ }^{\circ} \mathrm{C}$. The cells were then visualized using a microscope (ZEISS,Oberkochen, Germany).

In vivo imaging of SCC7 tumor. Animal experiments were conducted under protocols approved by Animal Care and Use Committee (CC/ACUCC) of Xiamen University. Subcutaneous sites of athymic nude mice (seven weeks old, female, 16-18 g) were injected a suspension of $4 \times 10^{6}$ SCC7 cells in PBS $(50 \mu \mathrm{L})$. When the tumor size (in the right leg region) reached average size of 120-150 $\mathrm{mm}^{3}$, mice were randomly allocated into three groups, (a) CPGA (10 mg GO $\left.\mathrm{kg}^{-1}\right)$ were injected into the tail vein of the mice. (b) CPG $\left(10 \mathrm{mg} \mathrm{GO} \mathrm{kg}^{-1}\right)$ were injected into the tail vein of the mice. (c) 30 min after MMP Inhibitor III injected, CPGA were injected via the tail vein. Fluorescent imagines were acquired at $0.15,1,4,6,12$ and $24 \mathrm{~h}$ after injectionusing IVIS Lumina II (Caliper Life Sciences, USA; Excitation Filter: 640 nm, Emission Filter: 700 nm).

When tumor volumes reached $100-150 \mathrm{~mm}^{3}$, mice were randomly allocated into four groups, CPGA (10 mg GO kg-1), CPG (10 mg GO kg ${ }^{-1}$ ), Au with the same concentration of gold on the 
surface of CPGA (10.4 mg Au kg${ }^{-1}$ ) and CP with the same NIR absorbance at $702 \mathrm{~nm}$ were injected into the tail vein of the tumor-bearing mice. After injection, PA images in the tumor sites were recorded on Endra Nexus128 (808 nm) at 0.15, 1, 4, 6 and $12 \mathrm{~h}$. At $24 \mathrm{~h}$ after one-dose injection, SCC7 tumors and normal organs (heart, liver, spleen, kidney, pancreas and muscle) were collected. Fluorescence images were captured with IVIS Lumina II and gold concentrations were recorded with inductively coupled plasma mass spectrometry (ICP-MS) (Agilent Technologies, Palo Alto, USA).

In vivo photothermal therapy. SCC7 cells $\left(4 \times 10^{6}\right.$ cells in $50 \mu \mathrm{L}$ of PBS $)$ were injected in the right leg of athymic nude mice (seven weeks old, 16-18 g). When the tumor size reached an average size of $60-70 \mathrm{~mm}^{3}$, CPGA (10 mg GO kg-1), CPG (10 mg GO kg $\left.{ }^{-1}\right), \mathrm{CP}$ and PBS were injected into the tail vein of the SCC7 tumor-bearing mice, respectively. At $4 \mathrm{~h}$ post-injection, tumors were irradiated with the $808 \mathrm{~nm}$ laser at a power density of $0.75 \mathrm{~W} \mathrm{~cm}^{-2}$ for $10 \mathrm{~min}$. Meanwhile, thermal images in tumors were recorded using a FLIR Ax5 camera and quantified by BM_IR software.

PTT therapies were further observed by recording tumor volumes and body weights. When the tumor size reached $60 \sim 70 \mathrm{~mm}^{3}$, SCC7 tumor-bearing mice were randomly divided into 8 groups: (a) CPGA without laser, (b) CPGA with laser, (c) CPG without laser, (d) CPG with laser, (e) CP without laser, (f) CP with laser, (g) PBS without laser and (h) PBS with laser. The b, d, f and $\mathrm{h}$ groupwere radiated with the same power density laser $\left(808 \mathrm{~nm}, 0.75 \mathrm{~W} \mathrm{~cm}^{-2}, 10 \mathrm{~min}\right)$. The tumor sizes were recorded by caliper every other day after treatment. Tumor volumes (V) were determined by the following equation: $\mathrm{v}=\mathrm{ab} / 2$, where $\mathrm{a}$ was the longer and $\mathrm{b}$ was the shorter diameter $(\mathrm{mm})$. The mouse body weights were measured with the same time points. After 14 days of treatment, the relative tumor volumes of all groups were calculated. Histological changes 
and apoptotic cells in tumor tissues and main organs were evaluated using hematoxylin and eosin (H\&E) staining.

Statistical Analysis. Results were expressed as mean and SD. Two-tailed paired and unpaired Student's t tests were used to determine differences within groups and between groups, respectively. $P$ value $<0.05$ was considered statistically significant.

\section{Results and Discussion}

\section{Preparation and characterization of hybrid GO/Au activatable probe (CPGA)}

In this study, MMP-14 sensitive substrate peptide with the sequence of Gly-Ala-Thr-ArgLeu-Phe-Gly-Ile-Arg-Gly was first synthesized and labeled with a near-infrared dye (NIR), Cy5.5, for in vivo fluorescent imaging. The product (CP) was purified by high performance liquid chromatography (HPLC) and confirmed by mass spectrometry (Figure S1 and S2, Supporting Information). To endow GO with enhanced photothermal conversion efficiency and improve GO fluorescent quenching effect, $\mathrm{Au}$ nanoparticles were seeded on the surface of GO. GA nanocomposite was prepared from an aqueous $\mathrm{GO}$ solution and $\mathrm{HAuCl}_{4}$ solution via oxidation-reduction reaction as reported[33]. Polyethylene glycol (PEG) with amine groups (molecular weight: $2 \mathrm{kDa}$ ) on both side was used to modify $\mathrm{CP}$, so that CP-PEG-amine can be further conjugated on to GO in GA complex, forming final production (CPGA). The successful construction of final GA hybrid nanocomplex, CPGA, was confirmed by transmission electron microscopy (TEM) and atomic force microscope (AFM) (Figure 1 and Figure S3). As shown in Figure 1a and Figure S3, a large amount of gold nanoparticles at diameters of $15 \pm 1.6 \mathrm{~nm}$ were

evenly grown on the surface of GO. AFM images also revealed that CPGA had a thickness of about $15 \mathrm{~nm}$ due to the planting of Au nanoparticles on GO surface and a diameter of about 230 
$\mathrm{nm}$ (Figure S3). In the consideration of GO sheet dimension $(1 \mathrm{~nm})$, the thickness increase of CPGA was mainly attributed to the formation gold nanoparticles on the surface of GO. The preparations of CPGA were also verified by UV-visible-near infrared (UV-vis-NIR) absorbance spectrum. A peak at $535 \mathrm{~nm}$ was observed in UV-vis-NIR absorbance spectra of CPGA, indicating the successful conjugation of Au particles onto GO (Figure 1b). Additionally, an absorbance peak for Cy5.5 at $700 \mathrm{~nm}$ was observed in CPGA complex. The red-shifted absorption of Cy5.5 was caused by the strong interaction between the Cy5.5 and GO[34, 35]. After CP conjugation onto GA, the fluorescent signals from Cy5.5 were significantly quenched due to the fluorescent resonance energy transfer (FRET) between GO and Cy5.5 and SPR effect between Au nanoparticles and Cy5.5. It has to point out that there is a shoulder for all phantoms absorbance spectrum, which was caused by the noise of instrument as ultrapure water also showed a similar peak at around $950 \mathrm{~nm}$ (Figure S5). For the best quenching effect, the fluorescent recovery folds were calculated by comparison of fluorescent signals before and after CPGA incubation with MMP14. Different amount of $\mathrm{CP}$ was conjugated onto GA and the fluorescent signal recovery fold was calculated as shown in Figure 1c. The fluorescent signals were found increased upto $11.78 \pm 0.38$ when the ratio between $\mathrm{CP}$ and GA reaches to 5:1. The quenching efficiency of CPGA in water solution was approximately 95\% upon $675 \mathrm{~nm}$ laser excitation, while the quenching efficiency of CPG alone was approximately $84 \%$, implying that CPGA was more sensitive to MMP-14 than either CP or CPG. Taken these results together, we concluded that CPGA was successfully prepared. We then examined the stability of CPGA by incubating it with ultrapure water, phosphate buffer (PBS), fetal bovine serum (FBS), and cell culture medium (Figure S4). We observed ideal dispersion and stability after $24 \mathrm{~h}$ incubation in mouse blood serum at room temperature, which were confirmed by UV-vis-NIR spectrum 
(Figure 1d) and no obvious absorbance changes were found. Zeta potential of CPGA was further measured $(-24.6 \pm 1.93 \mathrm{mV})$ and verified that CPGA was stable (Figure S4).

\section{MMP-14 sensitivity of CPGA}

Next, to access the target response of CPGA, fluorescent signal recovery of CPGA against activated MMP-14 was evaluated by incubation CPGA with $40 \mathrm{nM}$ MMP-14 in the presence or absence of a broad spectrum MMP inhibitor III (MMPI-III) in TCNB buffer. Fluorescence emission signals recovery of CPGA was monitored by spectrofluorometer continuously for 1 hour. As shown in Figure 2a, fluorescence emission signals of CPGA gradually increased and peaked at $50 \mathrm{~min}$ after incubation. A proportional relationship between recovered NIR fluorescent signals and CPGA concentrations were presented as in Figure $\mathbf{2 b}$. The spectrofluorometer plots clearly indicated that MMP-14 could activate NIR fluorescent signals of CPGA over time without MMP inhibitor (MMPI). The activation of CPGA could be inhibited in the presence of MMPI, supporting the selectivity of MMP-14 against CPGA. These results indicated that CPGA was responsive to MMP-14 and can be used for MMP-14-overexpressed tumor optical detection. In the view of the strong optical absorbance of both GO and Au particles, photoacoustic (PA) effect of the hybrid CPGA was checked. As expected, stronger PA signals were observed with the increase of CPGA concentrations (Figure 2c). A proportional relationship was found between concentration of CPGA and PA intensity as shown in Figure 2d. These data clearly demonstrated that CPGA had enhanced PA signals as compared to Au, CPG or $\mathrm{CP}$ alone at the same concentration, which could potentially allow precise PA analysis of CPGA distribution in vivo.

\section{In vitro photothermal ablation test of CPGA}


Furthermore, we evaluated whether the enhanced photothermal effects of CPGA, CPG, Au, and $\mathrm{CP}$ extended to in vitro studies under laser irradiation. Temperature changes of CPGA, CPG, $\mathrm{Au}$ and $\mathrm{CP}$ at the same concentration were monitored under NIR laser radiation at a low density $\left(\lambda=808 \mathrm{~nm}, 0.3 \mathrm{~W} \mathrm{~cm}^{-2}, 10 \mathrm{~min}\right)$ and recorded by using an infrared (IR) thermal camera. CPGA exhibited significant temperature increment $\left(\Delta \mathrm{T}=23.00^{\circ} \mathrm{C}\right)$, which was much higher than that of $\mathrm{CPG}\left(\Delta \mathrm{T}=16.97^{\circ} \mathrm{C}\right), \mathrm{Au}\left(\Delta \mathrm{T}=8.70^{\circ} \mathrm{C}\right)$ or $\mathrm{CP}\left(\Delta \mathrm{T}=1.57^{\circ} \mathrm{C}\right)$ alone (Figure 3a, b), suggesting that CPGA with the strong NIR absorption had improved photothermal effect. Escalating concentrations of CPGA or laser power yielded high temperature increases as shown in Figure S6. No obvious temperature elevation was observed when CP was exposed to the same laser irradiation. These results suggested that CPGA presented excellent photothermal conversion efficiency under the NIR radiation and was a promising PTT agent. To confirm the excellent photothermal conversion efficiency of CPGA, in vitro photothermal effect and cytotoxicity of CPGA were investigated by MTT assay and Calcein AM/propidium iodide (PI) live/dead staining. Concentration of CPGA, CPG and Au were adjusted to make sure they contains same amount of Go $\left(100 \mu \mathrm{g} \mathrm{mL}^{-1}\right)$ or $\mathrm{Au}\left(104 \mu \mathrm{g} \mathrm{mL}^{-1}\right)$ by measuring their absorbance. Cell viability of SCC7 cells was examined with or without $808 \mathrm{~nm}$ laser exposure $\left(0.5 \mathrm{~W} \mathrm{~cm}^{-2}\right.$ for $10 \mathrm{~min}$, Figure 3c). We found that CPGA and CPG exhibited inappreciable cytotoxicity at different concentrations in the absence of laser illustration. However, after $808 \mathrm{~nm}$ laser irradiation, over $80 \%$ tumor cells were killed in CPGA group, and only $56 \%$ of cells were dead in CPG in the laser-treated group. The photothermal effects were further distinguished by using Calcein AM/ PI co-staining (Figure 3d). In CP and PBS groups, almost all the SCC7 cells were alive regardless of NIR laser irradiation (Figure S7), while only a few SCC7 cells in CPG group were dead upon laser irradiation, indicating that CP and PBS did not have obvious cytotoxicity. On the contrary, 
most SCC7 cells in CPGA group were killedafter laser illumination, while minimal cell death was observed in cells treated with CPGA without laser irradiation. These results demonstrated that CPGA was capable of efficiently killing cancer cells by enhanced photothermal effects and was not cytotoxic without laser irradiation.

\section{In vivo photoacoustic and fluorescence imaging of CPGA}

Before commencing precise image-guided photothermal treatment of tumors in vivo, we first utilized the optical/PA properties of CPGA to analyze its biodistribution and tumor accumulation. CPGA was intravenously injected into SCC7 tumor-bearing mouse model. The fluorescent signal recovery in tumor area was monitored under optical imaging instruments with specific filter (ex/em: 670/690 nm) at indicated times in Figure 4a. A gradual increased fluorescence signal was observed which peaked at $6 \mathrm{~h}$ post-injection (p.i.). Tumor-to-muscle tissue $(\mathrm{T} / \mathrm{M})$ ratios were quantified and calculated as $1.33 \pm 0.03,1.71 \pm 0.23,2.59 \pm 0.46,3.64 \pm$ $0.51,2.84 \pm 0.41$ and $1.55 \pm 0.18$ at $0.1,1,4,6,12$, and $24 \mathrm{~h}$ p.i., respectively (Figure $4 \mathbf{b}$ ). To confirm the specificity, MMP inhibitor (Figure S8) was intratumorally injected 30 min before CPGA and very weak fluorescent signals were observed. Compared with NIR fluorescence imaging, photoacoustic (PA) imaging provided much higher spatial resolution images and therefore facilitated clarifying the local distribution of PA agents. As shown in Figure 4c, PA signals at the tumor site were obtained at different times and signals peaked at $6 \mathrm{~h}$, which were consistent with the results of fluorescence imaging. Clear accumulation of CPGA was observed in tumor blood vessels with time. Normalized PA signals of CPGA in the tumor sites were calculated as $1.42 \pm 0.32,1.95 \pm 0.25,2.5 \pm 0.27$ and $1.71 \pm 0.31$ at $1,4,6$, and $12 \mathrm{~h}$ p.i. (Figure 4d). PA signals of CPGA were higher than the control groups (CPG or Au) at the tumor sites, due to strong absorbance of GO and Au, implying great potential of CPGA as a PTT agent for tumor 
ablation. The major organs and tumor were harvested and subjected to fluorescent imaging after $24 \mathrm{~h}$ to investigate the biodistribution of CPGA. Strong fluorescent signals were found in tumor in CPGA-treated group compared to CPG-administrated group. The fluorescence recovery was significantly inhibited when MMP-14 activity was restrained in CPGA-treated group (Figure S9). The strong signals in the kidney were attributed likely to metabolism. As CPGA was stable in blood serum (Figure 1d), quantification of gold reflected the distribution of CPGA. As shown in Figure S10, Au concentration of CPGA at the tumor site and normal organs were also analyzed by inductively coupled plasma mass spectrometry (ICP-MS). We detected $7.06 \pm$ $0.49 \% \mathrm{ID} / \mathrm{g}$ of $\mathrm{Au}$ within the tumor site consistent with ex vivobiodistribution fluorescence imaging data.

\section{In vivo photothermal therapy}

To further research PTT in vivo, 40 SCC7 tumor-bearing mice were randomly divided into eight groups ( $\mathrm{n}=5$ /group): the CPGA group with laser irradiation (CPGA W/ laser, $\mathrm{n}=5$ ), and the CPGA group without laser irradiation (CPGA W/o laser, $n=5$ ); the CPG group with laser irradiation (CPG W/ laser, $\mathrm{n}=5$ ), and the $\mathrm{CPG}$ group without laser irradiation (CPG W/o laser, $\mathrm{n}$ $=5)$; the $\mathrm{CP}$ group with laser irradiation $(\mathrm{CP} \mathrm{W/} \mathrm{laser,} \mathrm{n}=5)$, and the $\mathrm{CP}$ group without laser irradiation (CP W/o laser, $\mathrm{n}=5$ ); the PBS group with laser irradiation (PBS W/ laser, $\mathrm{n}=5$ ), and the PBS group without laser irradiation (PBS W/o laser, $\mathrm{n}=5$ ). SCC7 tumor-bearing mice were subjected to the same exposure time $(10 \mathrm{~min})$ and laser density $\left(808 \mathrm{~nm}, 0.75 \mathrm{~W} \mathrm{~cm}^{-2}\right)$ in every treatment group. Based on in vivo fluorescence and photoacoustic imaging of CPGA results, the maximum accumulation of CPGA in the tumor site was at $6 \mathrm{~h}$ p.i. Therefore, tumors on the right legs were exposed with $808 \mathrm{~nm}$ laser $\left(0.75 \mathrm{~W} \mathrm{~cm}^{-2}\right)$ for 10 min at $6 \mathrm{~h}$ p.i., and temperature changes at the tumor site were recorded using an infrared (IR) thermal camera. As shown in 
Figure 5a and $\mathbf{5 b}$, the tumor temperature of CPGA group increased rapidly from $33.8^{\circ} \mathrm{C}$ to $49.7^{\circ} \mathrm{C}\left(\Delta \mathrm{T}=15.9^{\circ} \mathrm{C}\right)$. However, tumor temperature was not significantly changed in three other groups: the $\mathrm{CPG}$ group $\left(\Delta \mathrm{T}=9.6^{\circ} \mathrm{C}\right)$, the $\mathrm{CP}$ group $\left(\Delta \mathrm{T}=8.1^{\circ} \mathrm{C}\right)$ and the $\mathrm{CPG}$ group $(\Delta \mathrm{T}=5.6$ $\left.{ }^{\circ} \mathrm{C}\right)$. After treatment, tumor volumes and body weights were measured and recorded every other day. No obvious body weight changes were found (Figure 5d). In the CPGA W/ laser irradiation group (808 nm, $0.75 \mathrm{~W} \mathrm{~cm}^{-2}, 10 \mathrm{~min}$ ), SCC7 tumors were absolutely eliminated within 14 days, and tumor recurrence were not seen during our study (Figure 5e). Tumor growth in CPGadministrated groups were found inhibited in the first 2 days after treatment then continued to grow after that, indicating that the tumor ablation effect was not significant using single composition (graphene only) compared to hybrid composition (GA) under the same laser irradiation conditions. SCC7 tumor growth in CP W/ laser groups were slightly repressed likely due to Cy5.5 dye generation of singlet oxygen $\left({ }^{1} \mathrm{O}_{2}\right)$ under laser irradiation to kill tumor cells. It has to note that there may certain amount of SO generate from CPGA under laser irradiation. We therefore evaluated the ${ }^{1} \mathrm{O}_{2}$ generation ability by a commercialized kit. The kit contains 2', 7' Dichlorodihydrofluorescin diacetate (DCFH-DA) and is originally non-fluorescent. DCFH-DA will be converted into 2',7'-dichlorodihydrofluorescein (DCFH) by cellular esterase and become fluorescent after ${ }^{1} \mathrm{O}_{2}$ oxidize it into 2',7'-dichlorofluorescein (DCF) (ex/em: 488/525 nm). Fluorescent intensity is considered proportional to the amount of ROS [36]. As shown in Figure S11, Cy5.5 at high concentration indeed possessed ability to generate of ROS under laser irradiation. However, compared to widely used photosensitizer, for example Ce6, ROS generated by CPGA was not significantly high. According to Figure S10, less than 10\% of CPGA (less than $40 \mu \mathrm{g}$ of CPGA) is accumulated in tumor area, which may not be able to generate enough ROS for tumor ablation compared to typical photosensitizer (Figure S11). To this end, we considered 
that the PDT treatment of tumor is less and majority of tumor is ablated by PTT in our study. To confirm the effective ablation of tumor, tumor tissues and normal organs of mice in each group were collected at 3 days post treatment with/without laser irradiation and subjected to histological examination (Figure 5f and 6a). Obvious tumor tissues damage was found in the CPGA W/ laser group. Not apparent abnormality was found in the liver, spleen, kidney, heart, and lung (Figure 6b) for all groups. Our data verified that CPGA was notably effective in ablating tumor growth at low power laser illumination and was not toxic to normal organs, suggesting that CPGA can serve as an agent for optical/PA image-guided tumor photothermal therapy with great clinic translational potency.

\section{Conclusion}

In summary, a novel MMP14-activatable nanoprobe, CPGA, was successfully synthesized in our study. By chemically conjugating with Cy5.5-peptide-PEG, GO/Au maintained satisfactory stability and enhanced quenching effect. It was noted that this graphene/gold-based probe exhibited stronger NIR absorbance and higher photoconversion efficiency for multimodality fluorescence for photoacoustic image-guided enhanced photothermal therapy of SCC7 tumors. Compared with the controls, CPGA possessed high tumor accumulation and significantly enhanced fluorescence and photoacoustic signals for in vivo imaging. More importantly, dual modal image-guided PTT by CPGA demonstrated excellent SCC7 tumor ablation response at low laser radiation $(0.75 \mathrm{~W} \mathrm{~cm}-2$ for $10 \mathrm{~min})$ without detectable toxicity and side effects. Overall, our results highlight CPGA nanocomposites with fluorescence and photoacoustic imaging features as multifunctional probes for image-guided phototherapy.

Conflict of Interest: The authors declare no competing financial interest. 
Acknowledgement: This work was supported by National Science Foundation of China (NSFC) (81571708, 81501506, 51373144 and 81201129), National High Technology Research and Development Program of China (863 Program) (No. 2014AA020708) and the Fundamental Research Funds for the Central Universities (No. 20720150064).

\section{REFERENCES}

\section{Uncategorized References}

[1] J.F. Lovell, C.S. Jin, E. Huynh, H. Jin, C. Kim, et al., Porphysome nanovesicles generated by porphyrin bilayers for use as multimodal biophotonic contrast agents, Nat Mater 10 (2011) 324332.

[2] P. Huang, J. Lin, X. Wang, Z. Wang, C. Zhang, et al., Light-triggered theranostics based on photosensitizer-conjugated carbon dots for simultaneous enhanced-fluorescence imaging and photodynamic therapy, Adv Mater 24 (2012) 5104-5110.

[3] J.F. Lovell, T.W. Liu, J. Chen, G. Zheng, Activatable photosensitizers for imaging and therapy, Chem Rev 110 (2010) 2839-2857.

[4] W.I. Choi, J.Y. Kim, C. Kang, C.C. Byeon, Y.H. Kim, et al., Tumor regression in vivo by photothermal therapy based on gold-nanorod-loaded, functional nanocarriers, ACS Nano 5 (2011) 1995-2003.

[5] S.M. Lee, H.J. Kim, Y.J. Ha, Y.N. Park, S.K. Lee, et al., Targeted chemo-photothermal treatments of rheumatoid arthritis using gold half-shell multifunctional nanoparticles, ACS Nano 7 (2013) 50-57.

[6] S. Goel, F. Chen, E.B. Ehlerding, W. Cai, Molecular imaging: intrinsically radiolabeled nanoparticles: an emerging paradigm, Small 10 (2014) 3824.

[7] V.C. Sanchez, A. Jachak, R.H. Hurt, A.B. Kane, Biological interactions of graphene-family nanomaterials: an interdisciplinary review, Chem Res Toxicol 25 (2012) 15-34.

[8] S. Gurunathan, J. Woong Han, E. Kim, D.N. Kwon, J.K. Park, et al., Enhanced green fluorescent protein-mediated synthesis of biocompatible graphene, J Nanobiotechnology 12 (2014) 41.

[9] T. Tian, X. Shi, L. Cheng, Y. Luo, Z. Dong, et al., Graphene-based nanocomposite as an effective, multifunctional, and recyclable antibacterial agent, ACS Appl Mater Interfaces 6 (2014) 8542-8548.

[10] S. Guo, D. Wen, Y. Zhai, S. Dong, E. Wang, Platinum nanoparticle ensemble-on-graphene hybrid nanosheet: one-pot, rapid synthesis, and used as new electrode material for electrochemical sensing, ACS Nano 4 (2010) 3959-3968.

[11] X. Shi, H. Gong, Y. Li, C. Wang, L. Cheng, et al., Graphene-based magnetic plasmonic nanocomposite for dual bioimaging and photothermal therapy, Biomaterials 34 (2013) 47864793. 
[12] K. Yang, L. Hu, X. Ma, S. Ye, L. Cheng, et al., Multimodal imaging guided photothermal therapy using functionalized graphene nanosheets anchored with magnetic nanoparticles, Adv Mater 24 (2012) 1868-1872.

[13] X. Yang, N. Zhao, F.J. Xu, Biocleavable graphene oxide based-nanohybrids synthesized via ATRP for gene/drug delivery, Nanoscale 6 (2014) 6141-6150.

[14] S. Goenka, V. Sant, S. Sant, Graphene-based nanomaterials for drug delivery and tissue engineering, J Control Release 173 (2014) 75-88.

[15] Z. Gan, X. Wu, M. Meng, X. Zhu, L. Yang, et al., Photothermal contribution to enhanced photocatalytic performance of graphene-based nanocomposites, ACS Nano 8 (2014) 9304-9310.

[16] K. Yang, L. Feng, X. Shi, Z. Liu, Nano-graphene in biomedicine: theranostic applications, Chem Soc Rev 42 (2013) 530-547.

[17] L.V. Wang, Multiscale photoacoustic microscopy and computed tomography, Nature photonics 3 (2009) 503-509.

[18] A. De La Zerda, C. Zavaleta, S. Keren, S. Vaithilingam, S. Bodapati, et al., Carbon nanotubes as photoacoustic molecular imaging agents in living mice, Nature nanotechnology 3 (2008) 557-562.

[19] A.J. Coughlin, J.S. Ananta, N. Deng, I.V. Larina, P. Decuzzi, et al., Gadolinium-conjugated gold nanoshells for multimodal diagnostic imaging and photothermal cancer therapy, Small 10 (2014) 556-565.

[20] L. Cheng, J. Liu, X. Gu, H. Gong, X. Shi, et al., PEGylated WS(2) nanosheets as a multifunctional theranostic agent for in vivo dual-modal $\mathrm{CT} /$ photoacoustic imaging guided photothermal therapy, Adv Mater 26 (2014) 1886-1893.

[21] C. Liang, S. Diao, C. Wang, H. Gong, T. Liu, et al., Tumor metastasis inhibition by imagingguided photothermal therapy with single-walled carbon nanotubes, Adv Mater 26 (2014) 56465652.

[22] D. Niu, X. Wang, Y. Li, Y. Zheng, F. Li, et al., Facile synthesis of magnetite/perfluorocarbon co-loaded organic/inorganic hybrid vesicles for dual-modality ultrasound/magnetic resonance imaging and imaging-guided high-intensity focused ultrasound ablation, Adv Mater 25 (2013) 2686-2692.

[23] S. Wang, Z. Dai, H. Ke, E. Qu, X. Qi, et al., Contrast ultrasound-guided photothermal therapy using gold nanoshelled microcapsules in breast cancer, European journal of radiology 83 (2014) 117-122.

[24] L. Zhang, S. Gao, F. Zhang, K. Yang, Q. Ma, et al., Activatable hyaluronic acid nanoparticle as a theranostic agent for optical/photoacoustic image-guided photothermal therapy, ACS Nano 8 (2014) 12250-12258.

[25] C.J. Shearer, A. Cherevan, D. Eder, Application and future challenges of functional nanocarbon hybrids, Adv Mater 26 (2014) 2295-2318.

[26] T. Ji, Y. Zhao, Y. Ding, G. Nie, Using functional nanomaterials to target and regulate the tumor microenvironment: diagnostic and therapeutic applications, Adv Mater 25 (2013) 35083525 .

[27] X. Lin, Xie, J., Zhu, L., Lee, S., Niu, G., Ma, Y., Kim, K., Chen, X., Hybrid ferritin nanoparticles as activatable probes for tumor imaging, Angew Chem Int Ed Engl 50 (2011) $1569-1572$.

[28] L. Zhu, J. Xie, M. Swierczewska, F. Zhang, Q. Quan, et al., Real-time video imaging of protease expression in vivo, Theranostics 1 (2011) 18-27.

[29] E. Morales-Narvaez, A. Merkoci, Graphene oxide as an optical biosensing platform, Adv 
Mater 24 (2012) 3298-3308.

[30] X.P. He, Y. Zang, T.D. James, J. Li, G.R. Chen, Probing disease-related proteins with fluorogenic composite materials, Chem Soc Rev 44 (2015) 4239-4248.

[31] B. Dubertret, M. Calame, A.J. Libchaber, Single-mismatch detection using gold-quenched fluorescent oligonucleotides, Nat Biotechnol 19 (2001) 365-370.

[32] L. Zhu, H. Wang, L. Wang, Y. Wang, K. Jiang, et al., High-affinity peptide against MT1MMP for in vivo tumor imaging, J Control Release 150 (2011) 248-255.

[33] M.K. Chuang, S.W. Lin, F.C. Chen, C.W. Chu, C.S. Hsu, Gold nanoparticle-decorated graphene oxides for plasmonic-enhanced polymer photovoltaic devices, Nanoscale 6 (2014) 1573-1579.

[34] J. Huang, C. Zong, H. Shen, Y.H. Cao, B. Ren, et al., Tracking the intracellular drug release from graphene oxide using surface-enhanced Raman spectroscopy, Nanoscale 5 (2013) 1059110598.

[35] X.F. Zhang, S.P. Liu, X.N. Shao, Noncovalent binding of xanthene and phthalocyanine dyes with graphene sheets: The effect of the molecular structure revealed by a photophysical study, Spectrochimica Acta Part a-Molecular and Biomolecular Spectroscopy 113 (2013) 92-99.

[36] R.P. Rastogi, S.P. Singh, D.P. Hader, R.P. Sinha, Detection of reactive oxygen species (ROS) by the oxidant-sensing probe 2 ',7'-dichlorodihydrofluorescein diacetate in the cyanobacterium Anabaena variabilis PCC 7937, Biochemical and Biophysical Research Communications 397 (2010) 603-607. 


\section{FIGURE LEGENDS}

Scheme 1. Schematic illustration of CPGA as theranostic complex for NIR fluorescence and PA image-guided enhanced photothermal therapy. a) Synthesis of hybrid nanocomplex, CPGA. b) In vivo applications of CPGA for enhanced tumor ablation.

Figure 1. Characterization of CPGA. a) TEM image of synthesized CPGA, showing the average particle size at about $230 \mathrm{~nm}$. b) UV-vis-NIR spectra of CPGA, CP, CPG, GA, GO and Au in water solution. c) Fluorescence recovery fold of CPGA at different ratios of CP and GA in a solution containing MMP-14. Means \pm SD $(n=3)$. d) UV-vis-NIR spectrum of CPGA in mouse blood serum over time. Inner photos was CPGA in mouse blood serum after 24 hours.

Figure 2. In vitro enzyme activity test and PA signals of CPGA. a) Fluorescence signal recovery of CPGA in the presence of MMP-14 at indicated time points. Cy5.5 (ex/em: 675/695 nm) signals were monitored and recorded. Inserts are fluorescent images of CPGA before and after MMP14 activation. b) Fluorescence intensity change with CPGA after incubation with different amount of MMP-14 $\left(\mathrm{pH}=7.5,37^{\circ} \mathrm{C}\right)$ at indicated time points. c) PA images of CPGA, CPG, $\mathrm{Au}$ 
and CP aqueous solution at $808 \mathrm{~nm}$. d) Standard curves of PA signals of CPGA, CPG, Au and CP solutions.

Figure 3. In vitro photothermal experiments. a) Real-time thermal imaging of CPGA, CPG, CP and $\mathrm{Au}$ aqueous solution under irradiation of an $808 \mathrm{~nm}$ NIR laser $\left(808 \mathrm{~nm}, 0.3 \mathrm{~W} \mathrm{~cm}^{-2}\right.$ ) for 10 min. b) In vitro temperature change curve of CPGA, CPG, CP and Au with laser irradiation (808 $\mathrm{nm}, 0.3 \mathrm{~W} \mathrm{~cm}^{-2}$ for $10 \mathrm{~min}$ ). c) Cell viability studies with CPGA, CPG and CP with and without laser irradiation $\left(808 \mathrm{~nm}, 0.5 \mathrm{~W} \mathrm{~cm}^{-2}\right.$ for $\left.10 \mathrm{~min}\right)$ on SCC7 cells. Error bars were based on standard deviations of three parallel samples. d) Fluorescence microscopy images of SCC7 cells were incubated by CPGA and CPG $(100 \mu \mathrm{g}$ GO mL-1) with and without laser irradiation $808 \mathrm{~nm}$ laser $0.75 \mathrm{~W} \mathrm{~cm} \mathrm{~cm}^{-2}$ for $5 \mathrm{~min}$. The cells are all co-stained by Calcein-AM (live: green) and Propidium Iodide (dead: red).

Figure 4. In vivo NIR fluorescence and PAT imaging. a) In vivo NIR fluorescent imaging of SCC7 tumor-bearing mice were taken at different times after intravenous injection of CPGA, CPGA with Inhibitor and CPG respectively. Arrows indicate the tumors location. b) Tumor/muscle (T/M) ratio of SCC7 tumor-bearing mouse model. c) In vivo PA imaging of blood vessels in the tumor sites at different time points after intravenous injection of CPGA, CPG and $\mathrm{Au}$ (the injected amount of CPGA and CPG were $10.0 \mathrm{mg} \mathrm{GO} \mathrm{kg-1;} \mathrm{the} \mathrm{injected} \mathrm{amount} \mathrm{of}$ CPGA and Au were $10.4 \mathrm{mg} \mathrm{Au} \mathrm{kg-1),} \mathrm{respectively.} \mathrm{d)} \mathrm{Photoacoustic} \mathrm{intensity} \mathrm{of} \mathrm{tumor} \mathrm{tissues}$ at different time points. $\mathrm{n}=3$ per group.

Figure 5. In vivo PTT. a) Thermal images of SCC7 tumor-bearing mice intravenously treated with CPGA, CPG, CP and PBS (the injected amount of CPGA and CPG were $10 \mathrm{mg} \mathrm{GO} \mathrm{kg}^{-1}$, CPGA, CPG and CP had the same content of Cy5.5, the tumor is located in the right leg) and exposure to $808 \mathrm{~nm}$ laser irradiation $\left(0.75 \mathrm{~W} \mathrm{~cm}^{-2}\right)$ for $10 \mathrm{~min}$ at $6 \mathrm{~h}$ post-injection. White circles 
indicates the location of tumors. b) Tumor heating curves of SCC7 tumor-bearing mice injected CPGA, CPG, CP and PBS at different time points. c) Tumor growth curves of different groups of SCC7 tumor-bearing mice after treatment. Tumor volumes were normalized to their initial size. Error bars represent the standard deviations of 5 mice per group. $\mathrm{P}<0.05$. d) Body weight curves of SCC7 tumor-bearing mice in different groups. The error bars represent the standard deviations of 5 mice per group. e) Typical photographs of SCC7 tumor- bearing mice at different days after CPGA treatment. f) H\&E stained tumor sections of SCC7 tumor-bearing mice in different groups at 3 days post treatment: CPGA w/ laser, $\mathrm{CPG}$ w/ laser, $\mathrm{CP}$ w/ laser and PBS w/ laser.

Figure 6. a) Representative photos of mice after different treatments. H\&E stained tumor sections collected from different groups of mice at indicated time points. Severely damaged tumor tissue was observed in CPGA treated group after irradiation, and slightly damaged tumor tissue were observed in CPG and CP after irradiation. (b) Histological images of major organs using H\&E staining. No noticeable abnormality was found in the heart, liver, spleen, lung, or kidney. 


\section{Scheme 1}

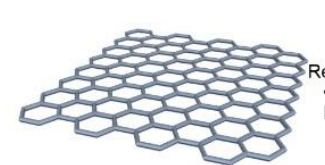

GO

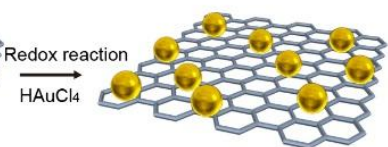

GO/Au (GA)
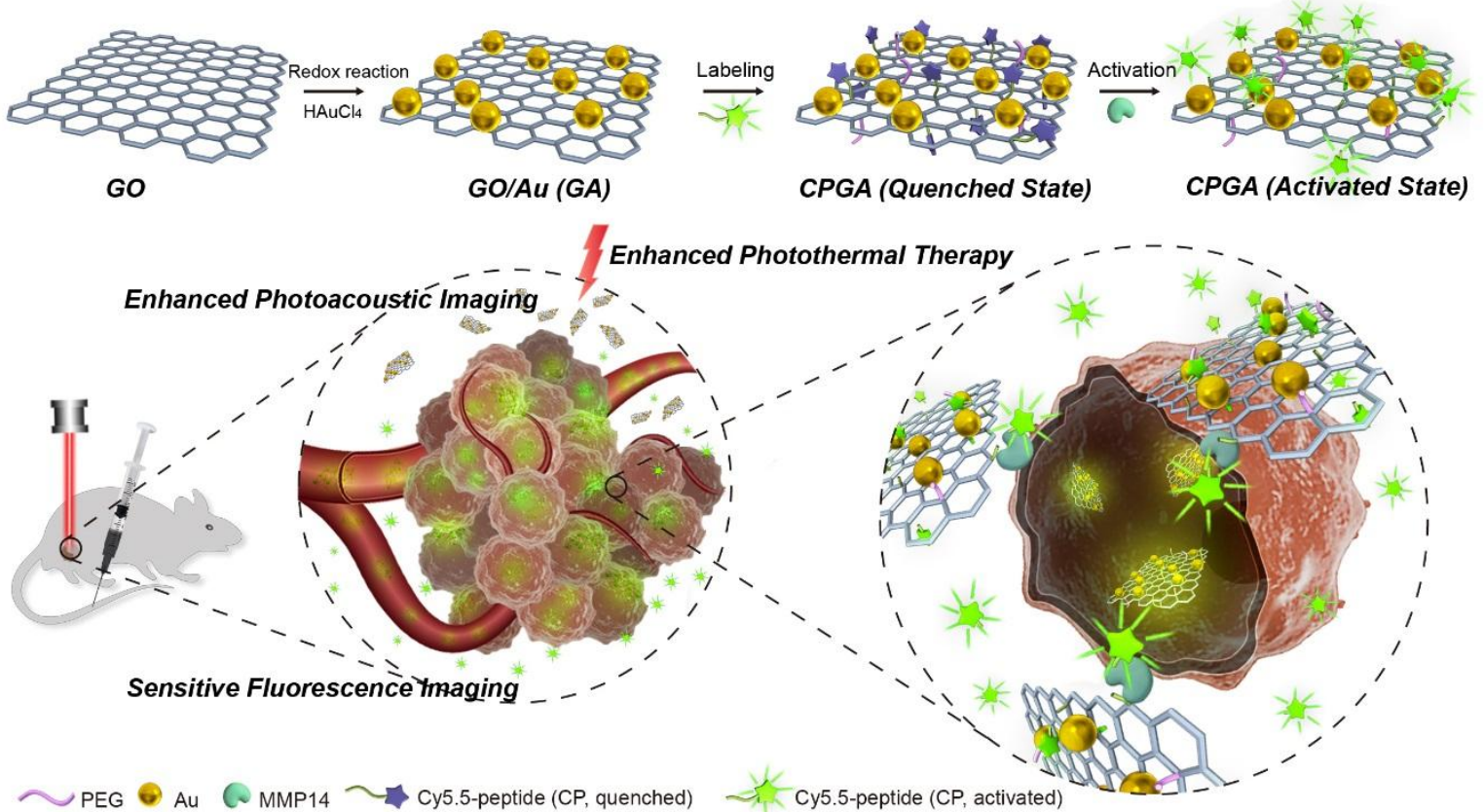
Figure 1.

a

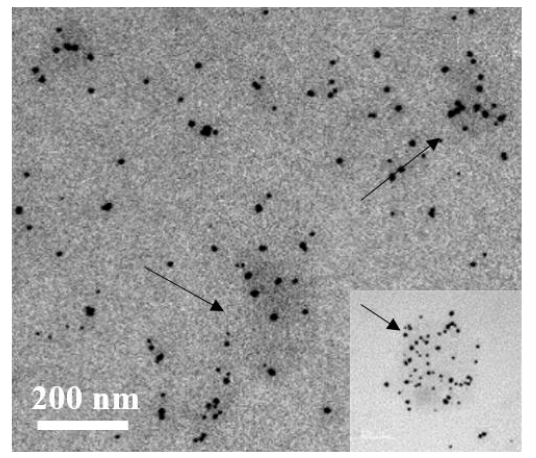

c

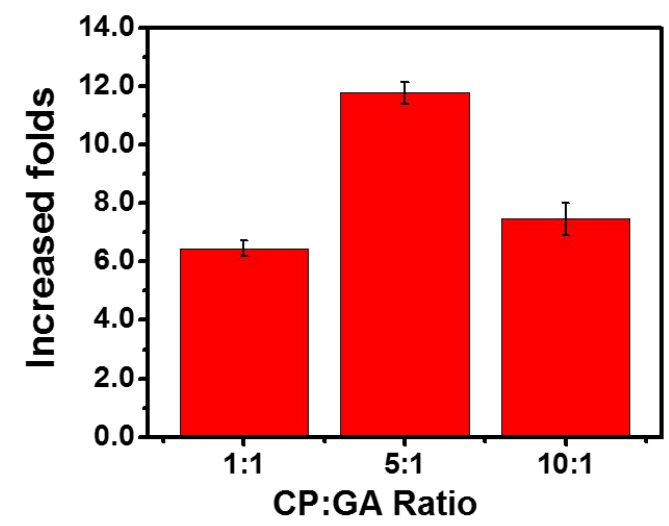

b

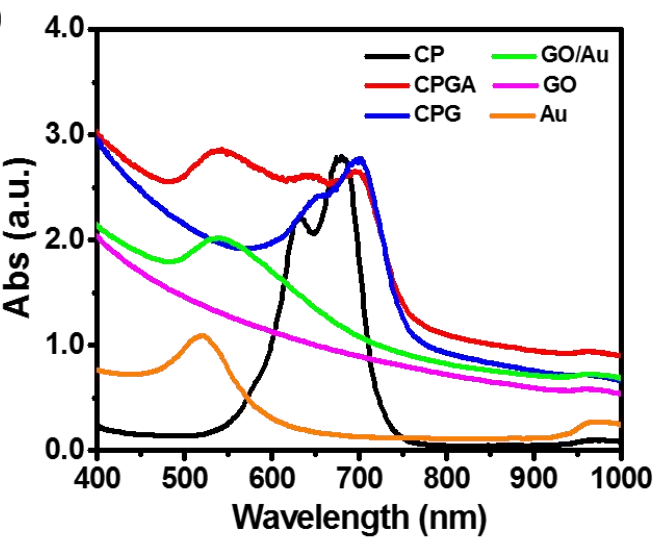

d

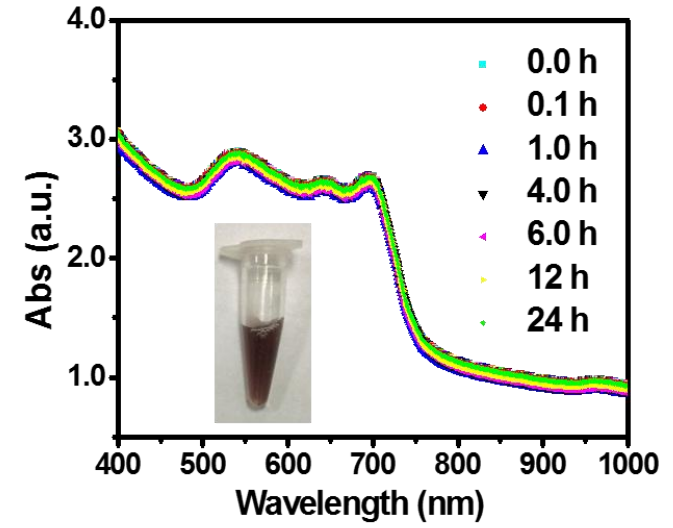


Figure 2.
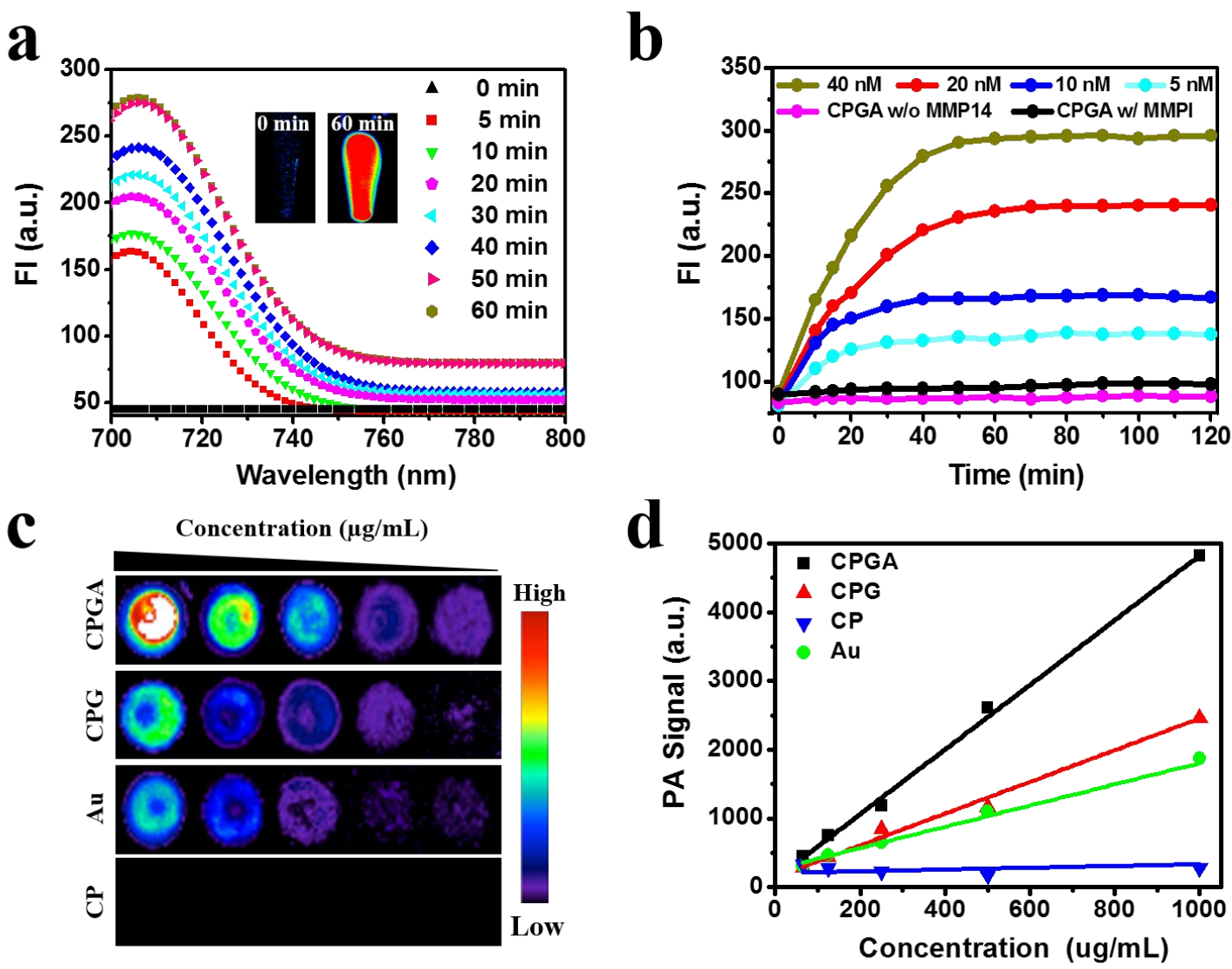
Figure 3

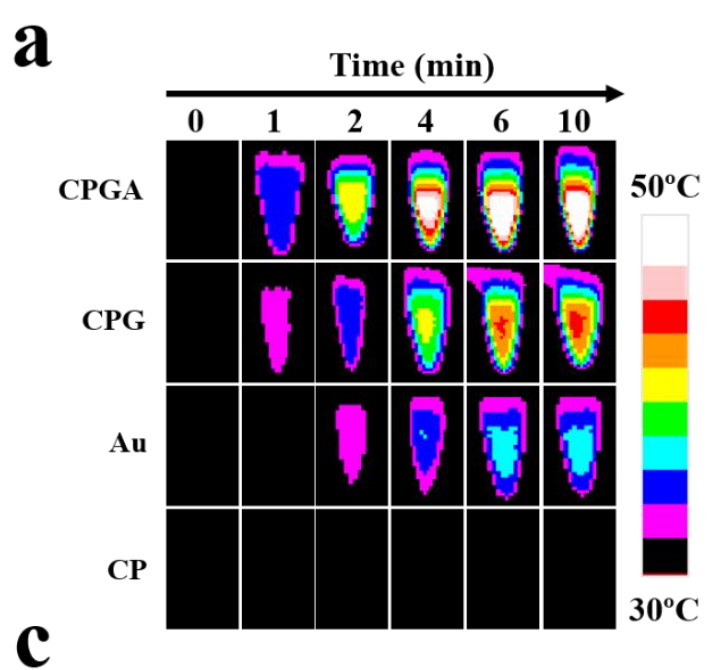

b
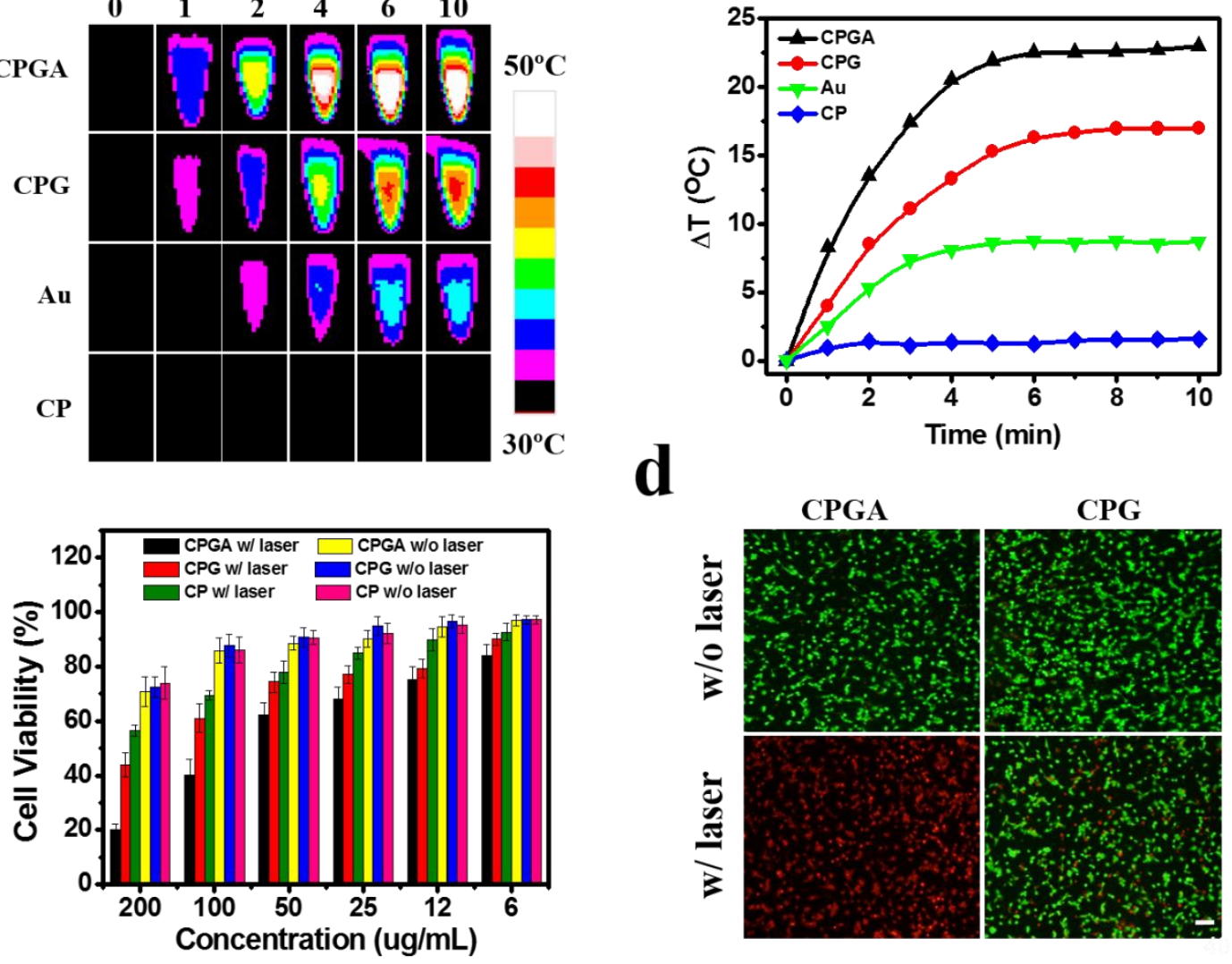
Figure 4.

a

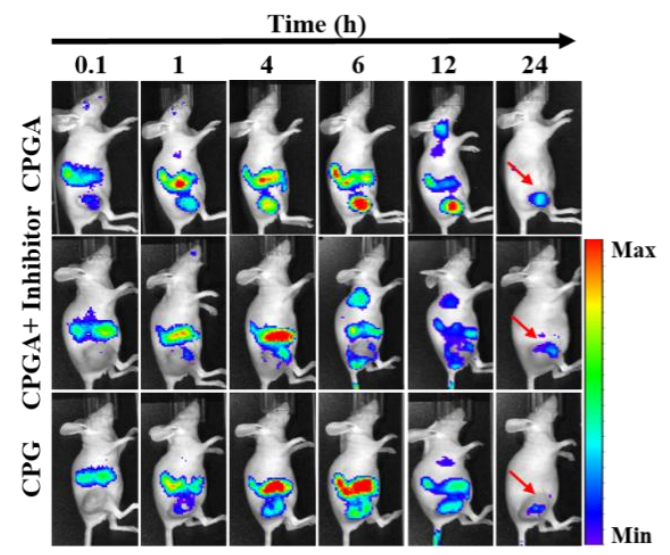

c

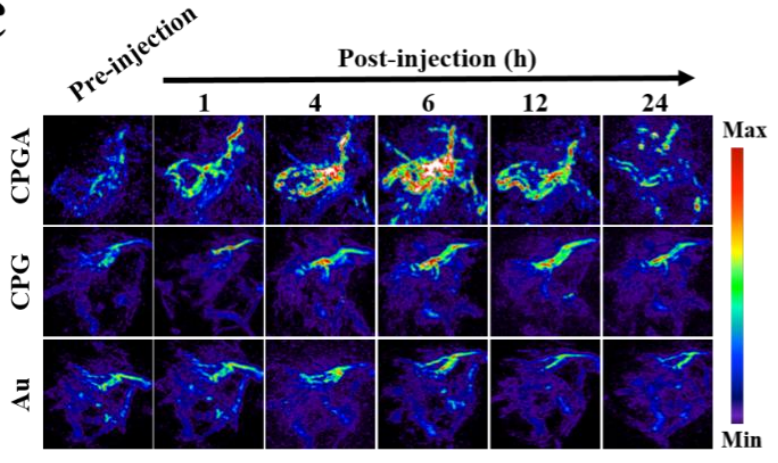

b

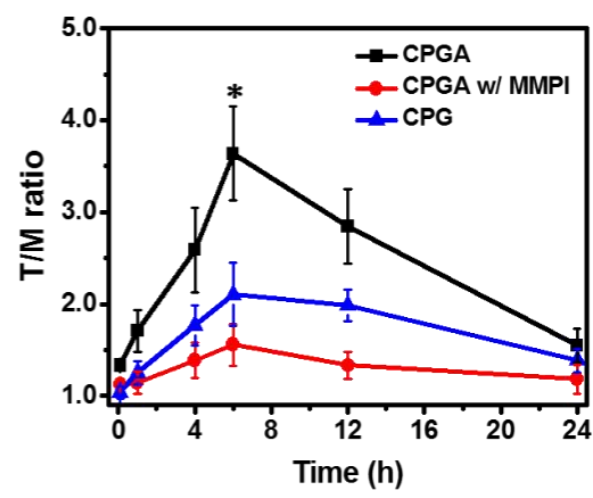

d

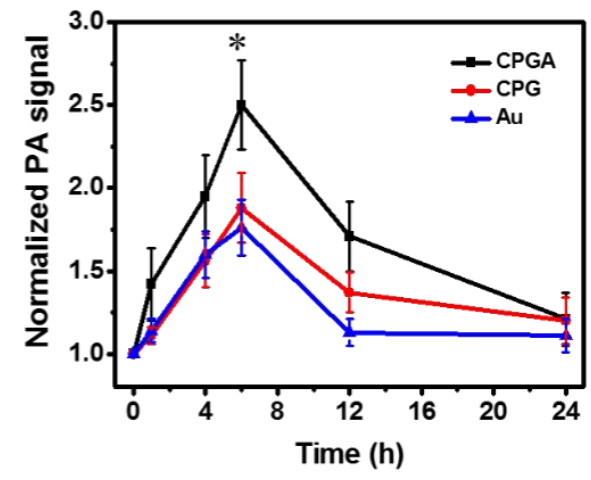


Figure 5.

a

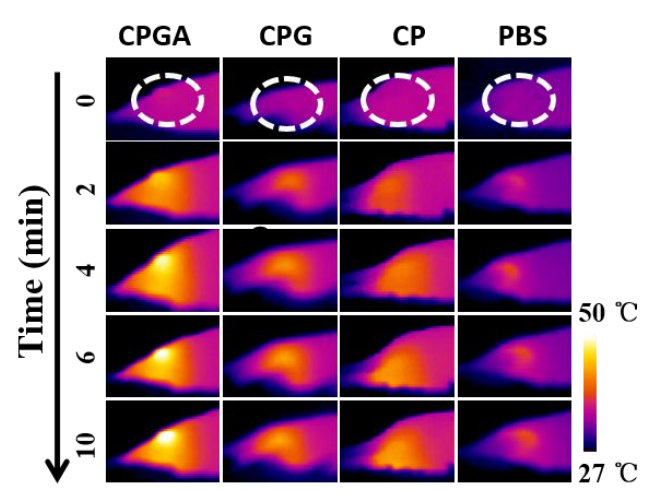

c

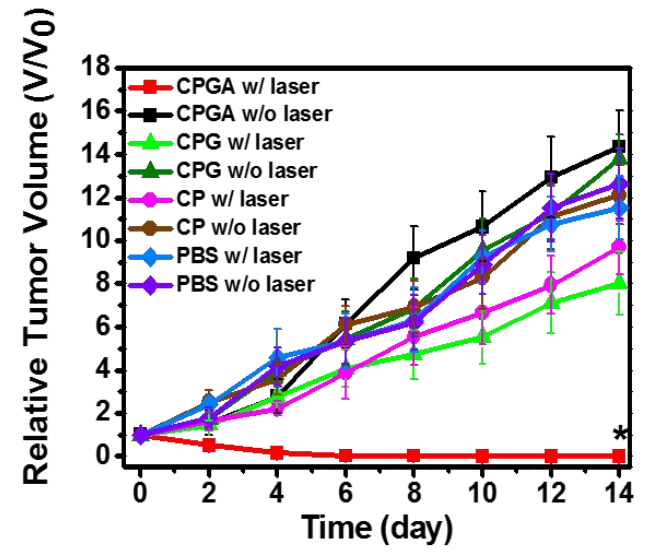

e

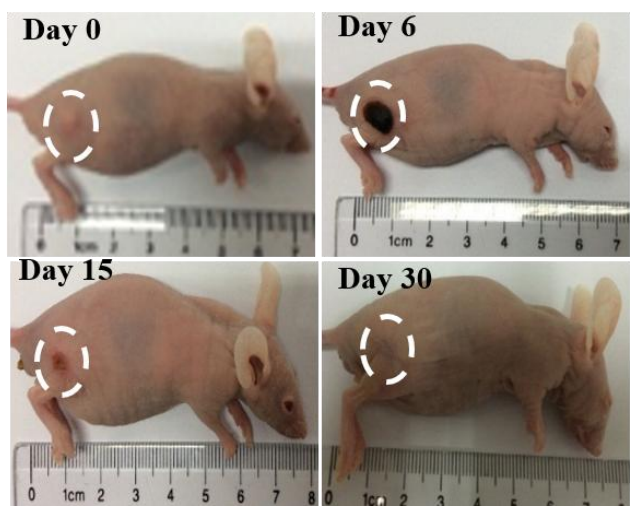

b

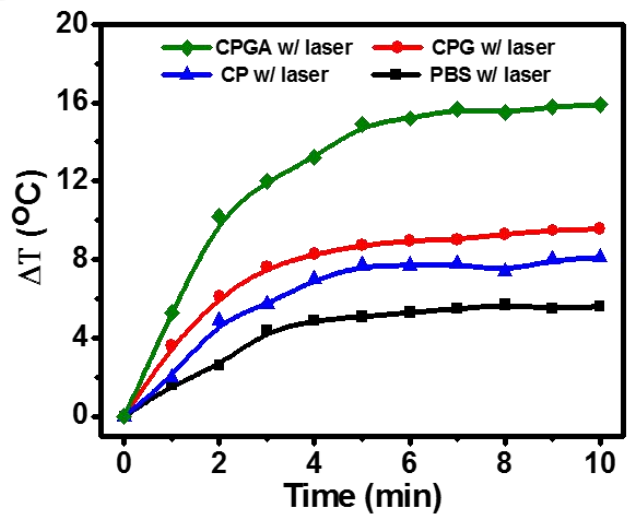

d

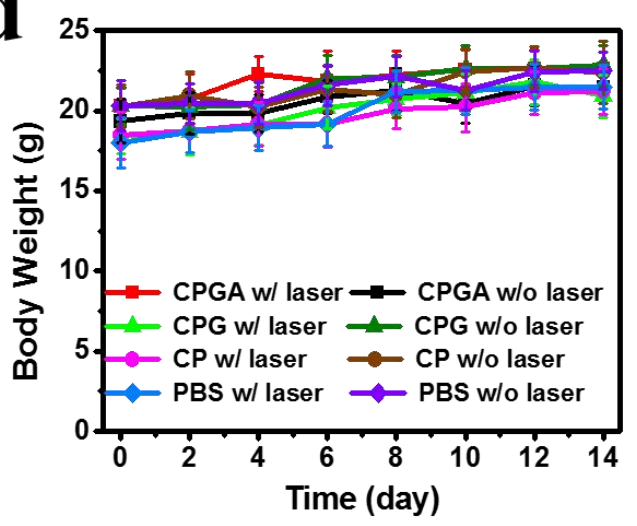

1

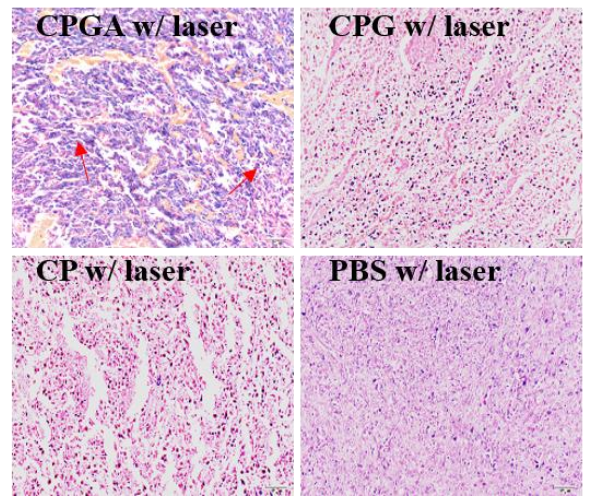


Figure 6.
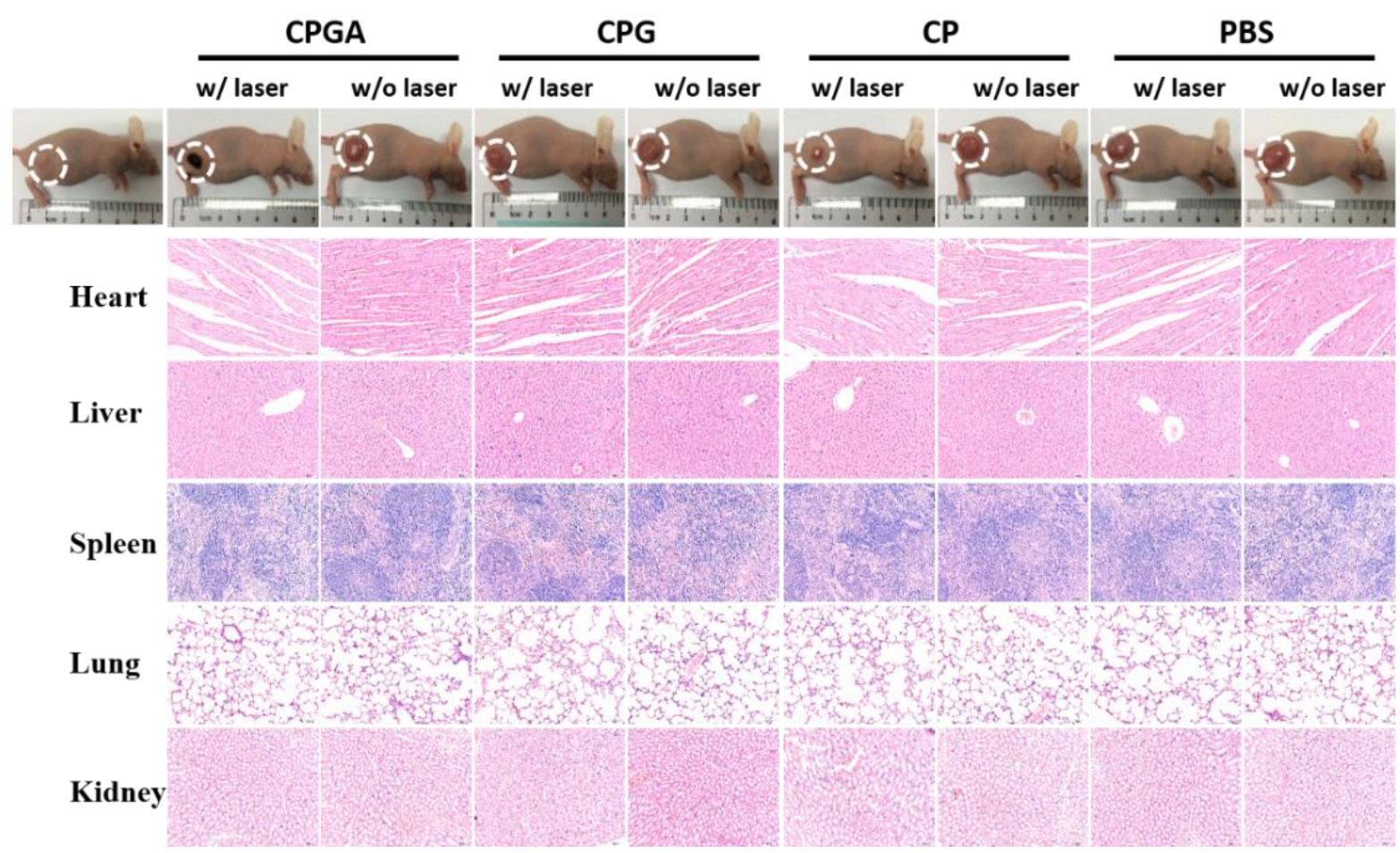